\title{
An online tool for predicting the prognosis of cancer patients with SARS-CoV-2 infection: a multi-center study
}

\author{
Congkuan Song ${ }^{1,2} \cdot$ Zhe Dong $^{1} \cdot$ Hongyun Gong ${ }^{3} \cdot$ Xiao-Ping Liu $^{4} \cdot$ Xiaorong Dong $^{5} \cdot$ Aifen Wang ${ }^{6} \cdot$ Yuan Chen ${ }^{7}$. \\ Qibin Song ${ }^{3} \cdot$ Weidong $\mathrm{Hu}^{1,2}$ (])
}

Received: 29 June 2020 / Accepted: 3 October 2020 / Published online: 11 October 2020

○) Springer-Verlag GmbH Germany, part of Springer Nature 2020

\begin{abstract}
Purpose During the 2019 coronavirus disease (COVID-19) pandemic, oncologists face new challenges, and they need to adjust their cancer management strategies as soon as possible to reduce the risk of SARS-CoV-2 infection and tumor recurrence. However, data on cancer patients with SARS-CoV-2 infection remains scarce.

Methods We conducted a retrospective study on 223 cancer patients with SARS-CoV-2 from 26 hospitals in Hubei, China. An individualized nomogram was constructed based on multivariate Cox analysis. Considering the convenience of the nomogram application, an online tool was also created. The predictive performance and clinical application of nomogram were verified by $\mathrm{C}$-index, calibration curve and decision curve analysis (DCA).

Results Among cancer patients with SARS-CoV-2, there were significant differences in clinical characteristics between survivors and non-survivors, and compared with patients with solid tumors including lung cancer, patients with hematological malignancies had a worse prognosis. Male, dyspnea, elevated PCT, increased heart rate, elevated D-dimers, and decreased platelets were risk factors for these patients. Furthermore, a good prediction performance of the online tool (dynamic nomogram: https://covid-19-prediction-tool.shinyapps.io/DynNomapp/) was also fully demonstrated with the C-indexes of 0.841 (95\% CI 0.782-0.900) in the development cohort and 0.780 (95\% CI 0.678-0.882) in the validation cohort.

Conclusion Overall, cancer patients with SARS-CoV-2 had unique clinical features, and the established online tool could guide clinicians to predict the prognosis of patients during the COVID-19 epidemic and to develop more rational treatment strategies for cancer patients.
\end{abstract}

Keywords Cancer $\cdot$ COVID-19 $\cdot$ SARS-CoV-2 $\cdot$ Nomogram $\cdot$ Prognosis

Congkuan Song, Zhe Dong and Hongyun Gong contributed equally to this study.

Electronic supplementary material The online version of this article (https://doi.org/10.1007/s00432-020-03420-6) contains supplementary material, which is available to authorized users.

Yuan Chen

chenyuan008@163.com

Qibin Song

qibinsong@whu.edu.cn

Weidong $\mathrm{Hu}$

huwd@whu.edu.cn

Extended author information available on the last page of the article

\section{Introduction}

Since the outbreak of coronavirus disease (COVID-19) in December 2019, the number of infected cases has been increasing, which has seriously affected the normal life of human beings. Recently, increased studies have suggested that cancer patients were more susceptible to SARSCoV-2 infection (Dai et al. 2020; Liang et al. 2020). Thus, oncologists face the new challenge during the COVID-19 pandemic. They need to adjust their cancer management strategies as soon as possible to reduce the risk of SARSCoV-2 infection and tumor recurrence without affecting the therapeutic effect. However, to date, data on SARS-CoV-2 infected cancer patients are still limited, and their prognosis is poorly understood. Given this, we performed a retrospective analysis based on the data on 223 cancer patients with SARS-CoV-2 infection from 26 clinical centers in Hubei 
province, China. In addition, we constructed an online nomogram to predict the prognosis of these patients, which might help clinicians develop more rational treatment strategies for cancer patients during the COVID-19 epidemic.

\section{Materials and methods}

\section{Study subject and ethical statements}

All cancer patients with SARS-CoV-2 infection were hospitalized in 26 hospitals in Hubei province, China from December 27, 2019 to March 19, 2020. A total of 296 patients were enrolled, of whom 62 patients lacking a SARSCoV-2 PCR test were excluded from this study. In addition, a one-year-old child was also excluded from this study. Eventually, 223 patients were selected in this study, who met the diagnostic criteria of the WHO interim guidance (https://www.who.int/publications-detail/clinical-manag ementof-severeacute-respiratory-infection-when-novelcoron avirus-(ncov)-infection-issuspected) and were confirmed by the nucleic acid test. This study was approved by the ethics committee of the National Cancer Center/Cancer Hospital, Chinese Academy of Medical Sciences and Peking Union Medical College (Approval No. 20/061-2257).

\section{Data collection and extraction}

The clinicopathological characters of 223 patients included in this study were collected from their medical records. General data included age, sex, BMI, smoking history, comorbidities (such as diabetes mellitus, arterial hypertension, etc.), vital signs, tumor types, dyspnea, fever and other symptoms, survival time and the interval to the negative conversion of SARS-Cov-2 nucleic acid test. Laboratory findings included procalcitonin (PCT), white blood cell count, neutrophil count, lymphocyte count, monocyte count, eosinophil count, basophil count, platelet count, aspartate aminotransferase (AST), alamine aminotransferase (ALT), $\mathrm{C}$ reactive protein (CRP), hypersensitive $\mathrm{C}$ reactive protein (hs-CRP), creatinine, serum amyloid A (SAA), creatine kinase isozyme (CK-MB), direct bilirubin, total bilirubin, prothrombin time (PT), activation of partial thrombin time (APTT) and D-dimer. Besides, treatment included antitumor therapy, antiviral therapy, antibacterial therapy, hormone therapy, and traditional Chinese medicine treatment.

\section{Statistical analysis}

Categorical variables were represented by a number (percentage). The continuous variables conforming to the normal distribution were represented by mean (standard deviation, $\mathrm{SD}$ ) otherwise by median (interquartile range, IQR).
The unpaired independent sample $t$ test was used for the continuous variables with homogeneous variances and normal distribution. Non-parametric tests (Mann-Whitney U and/or Kruskal-Wallis H) were used for those with uneven variances or non-normal distribution. The Chi-square test or Fisher's exact test was used to compare the categorical variables between groups, as appropriate. The nucleic acid test time of diagnosis was taken as the starting point of survival time, and the Kaplan-Meier method was used for survival analysis. In this study, all patients were randomly divided into a development cohort and a validation cohort at a ratio of 7:3. Based on the results of the univariate and multivariate Cox analysis in the development cohort, a nomogram was constructed with the "rms" package in R. To evaluate the prediction performance of the nomogram, we calculated the $\mathrm{C}$-indexes of the development cohort and validation cohort with bootstraps method. At the same time, calibration curves were also used to judge the consistency between the model prediction value and the actual observed value. In this study, all statistical analysis was carried out in R software (version 3.6.0). $P$ value $<0.05$ was considered statistically significant.

\section{Results}

\section{The clinicopathological features of SARS-Cov-2 infected cancer patients}

Of the 223 patients finally included in this study, 186 patients survived, and 37 patients were not (Table 1). 52.1\% of patients were male, $59.7 \%$ of patients presented dyspnea, and $75.7 \%$ of patients had a fever. Compared with survivors, non-survivors were older (67 years vs. 62.5 years), more male ( $70.3 \%$ vs. $48.4 \%)$, and more likely to have dyspnea (75.7\% vs. $66.7 \%)$. Moreover, non-survivors had a faster heart rate than survivors (100 bpm vs. $88 \mathrm{bpm}$ ). In the vast majority of laboratory findings, non-survivors had higher detection values, such as white blood cell count, neutrophil count, PCT, CRP, hs-CRP, direct bilirubin, PT, APTT, creatinine, CK-MB, and D-dimers. While platelet count and lymphocyte count were lower in the non-survivors, the differences were statistically significant (all $p<0.05$ ). Of these 223 patients, there were 82 patients with arterial hypertension and 32 patients with diabetes mellitus. The proportion of non-survivors with underlying diseases was higher than that of survivors, as shown in Figure S1. Among these 223 patients, 171 patients turned nucleic acid to be negative before the follow-up deadline, with a median time of 13 days. However, nucleic acid switching negative was not found in 37 non-survivors. Patients without nucleic acid negative conversion had more dyspnea than those with nucleic acid switching negative $(p<0.001)$. Similarly, patients without nucleic acid switching negative had higher PCT, HR, 
Table 1 Demographic characteristics of SARS-CoV-2-infected cancer patients in the entire cohort

\begin{tabular}{|c|c|c|c|c|}
\hline Variables & All patients $(n=223)$ & Survivors $(n=186)$ & Non-survivors $(n=37)$ & $p$ value \\
\hline Age (years) & $63(56,71)$ & $62.5(56,70)$ & $67(54.5,75)$ & 0.240 \\
\hline Sex & & & & 0.015 \\
\hline Female & 107 (47.9) & $96(51.6)$ & $11(29.7)$ & \\
\hline Male & $116(52.1)$ & $90(48.4)$ & $26(70.3)$ & \\
\hline BMI $\left(\mathrm{kg} / \mathrm{m}^{2}\right)$ & & & & 0.183 \\
\hline$<18.5$ & $13(5.8)$ & $12(6.5)$ & $1(2.7)$ & \\
\hline $18.5-24.9$ & $73(32.8)$ & $65(34.9)$ & $8(21.6)$ & \\
\hline$\geq 25$ & $30(13.5)$ & $26(14.0)$ & $4(10.8)$ & \\
\hline Unknown & $107(47.9)$ & $83(44.6)$ & $24(64.9)$ & \\
\hline Smoke & & & & 0.351 \\
\hline Never & $170(72.6)$ & $144(77.4)$ & $26(70.3)$ & \\
\hline Previous/present & $53(27.4)$ & $42(22.6)$ & $11(29.7)$ & \\
\hline Tumor types & & & & 0.001 \\
\hline Hematological malignancies & $15(6.7)$ & $7(3.7)$ & $8(21.6)$ & \\
\hline Lung cancer & $39(17.5)$ & $31(16.7)$ & $8(21.6)$ & \\
\hline Other solid tumors & $169(75.8)$ & $148(79.6)$ & $21(56.8)$ & \\
\hline Anti-tumor therapy & & & & 0.049 \\
\hline Continous & $126(56.5)$ & $111(59.7)$ & $15(40.5)$ & \\
\hline Discontinous & $30(13.4)$ & $21(11.3)$ & $9(24.4)$ & \\
\hline Unknown & $67(30.1)$ & $54(29.0)$ & $13(35.1)$ & \\
\hline Basic diseases * & & & & 0.608 \\
\hline Without & $105(47.1)$ & 89 (47.9) & $16(43.2)$ & \\
\hline With & $118(52.9)$ & $97(52.1)$ & $21(56.8)$ & \\
\hline Fever & & & & 0.687 \\
\hline Without & $54(24.3)$ & $46(24.7)$ & $8(21.6)$ & \\
\hline With & $169(75.7)$ & $140(75.3)$ & $29(78.4)$ & \\
\hline Dyspnea & & & & $<0.001$ \\
\hline Without & $90(40.3)$ & $62(33.3)$ & $9(24.3)$ & \\
\hline With & $133(59.7)$ & $124(66.7)$ & $28(75.7)$ & \\
\hline Other symptoms * & & & & 0.643 \\
\hline Without & $28(12.5)$ & $22(11.8)$ & $6(16.2)$ & \\
\hline With & $195(87.5)$ & $164(88.2)$ & $31(83.8)$ & \\
\hline PCT (ng/ml) & & & & $<0.001$ \\
\hline$\leq 0.5$ & $163(73.0)$ & $144(77.4)$ & $19(51.4)$ & \\
\hline$>0.5$ & $30(13.5)$ & $17(9.1)$ & $13(35.1)$ & \\
\hline Not application & $30(13.5)$ & $25(13.5)$ & $5(13.5)$ & \\
\hline Heart rate (bpm) & $88(78,100)$ & $86(77,97.25)$ & $100(88.5,112.5)$ & $<0.001$ \\
\hline $\mathrm{SBP}(\mathrm{mmHg})$ & $130(120,140)$ & $130(120,140.25)$ & $128(109,140.5)$ & 0.314 \\
\hline DBP (mmHg) & $78.33(11.128)$ & $77.8602(11.132)$ & 80.703 (10.952) & 0.156 \\
\hline Respiratory rate (braths/min) & $20(19,22)$ & $20(19,22)$ & $20(20,23)$ & 0.152 \\
\hline Temperature $\left({ }^{\circ} \mathrm{C}\right)$ & $36.7(36.5,37.5)$ & $36.7(36.5,37.5)$ & $36.9(36.4,38.05)$ & 0.703 \\
\hline WBC count (10E9/L) & $5.28(4.05,7.18)$ & $5.025(4.005,6.423)$ & $7.23(5.035,11.785)$ & $<0.001$ \\
\hline Neutrophil count (10E9/L) & $3.64(2.59,5.45)$ & $3.42(2.563,4.793)$ & $6.62(3.25,10.345)$ & $<0.001$ \\
\hline Lymphocyte count (10E9/L) & $0.90(0.61,1.42)$ & $0.95(0.658,1.433)$ & $0.69(0.495,1.125)$ & 0.024 \\
\hline Platelet count (10E9/L) & $188(117,249)$ & $196.5(130.75,260.25)$ & $142(51,203.5)$ & $<0.001$ \\
\hline Monocyte count (10E9/L) & $0.43(0.30,0.62)$ & $0.42(0.3,0.59)$ & $0.64(0.28,1.005)$ & 0.041 \\
\hline Eosinophils count $(10 E 9 / \mathrm{L})$ & $0.03(0,0.09)$ & $0.05(0.01,0.09)$ & $0(0,0.02)$ & $<0.001$ \\
\hline Basophil count (10E9/L) & $0.01(0.01,0.02)$ & $0.01(0.01,0.02)$ & $0.01(0,0.03)$ & 0.739 \\
\hline $\operatorname{ALT}(\mathrm{U} / \mathrm{L})$ & $22(13,36)$ & $23(14,37)$ & $18(10.5,26.5)$ & 0.068 \\
\hline AST (U/L) & $28(18,39)$ & $26.5(18,38)$ & $35(20,51)$ & 0.061 \\
\hline
\end{tabular}


Table 1 (continued)

\begin{tabular}{|c|c|c|c|c|}
\hline Variables & All patients $(n=223)$ & Survivors $(n=186)$ & Non-survivors $(n=37)$ & $p$ value \\
\hline Creatinine (umol/L) & $65(52.7,85)$ & $63.5(52.9,81.025)$ & $94.1(51.85,120.5)$ & 0.018 \\
\hline $\mathrm{CRP}(\mathrm{mg} / \mathrm{L})$ & $28.85(7.7,77.925)(n=176)$ & $25.4(5.225,55.5)(n=144)$ & $94.8(39.45,139.525)(n=32)$ & $<0.001$ \\
\hline $\mathrm{SAA}(\mathrm{mg} / \mathrm{L})$ & $148.39(26.8,300)(n=35)$ & $128.21(16.25,300)(n=25)$ & $183.195(79.483,300)(n=10)$ & 0.811 \\
\hline hs-CRP (mg/L) & $27.4(5,71.9)(n=159)$ & $21.965(5,59.3)(n=130)$ & $84.1(55.22,141.15)(n=29)$ & $<0.001$ \\
\hline Total bilirubin (umol/L) & $11.1(8,14.6)$ & $11(7.8,14.6)$ & $12.4(9.35,15.15)$ & 0.185 \\
\hline Direct bilirubin (umol/L) & $3.9(2.8,5.5)$ & $3.7(2.6,5.3)$ & $5.1(3.775,7.8)$ & $<0.001$ \\
\hline PT (s) & $12.9(11.9,14.0)(n=219)$ & $12.8(11.9,13.6)(n=182)$ & $14.4(12.3,15.75)$ & $<0.001$ \\
\hline APTT (s) & $34.6(29.1,39.7)(n=216)$ & $33.9(28.8,38.65)(n=181)$ & $37.8(30.7,46.2)(n=35)$ & 0.010 \\
\hline Creatine kinase-MB (U/L) & $1.12(0.525,4.525)(n=153)$ & $1.03(0.5,3.745)(n=133)$ & $3.645(1.025,14.738)(n=20)$ & 0.020 \\
\hline D-dimer (mg/L) & $0.837(0.37,2.14)$ & $0.69(0.329,1.9)$ & $1.63(0.465,5.865)$ & 0.001 \\
\hline Nucleic acid negative time & $13(9,22)$ & $13(9,22)$ & & \\
\hline Antiviral therapy & & & & 0.385 \\
\hline No & $19(8.5)$ & $14(7.5)$ & $5(13.5)$ & \\
\hline Yes & $204(91.5)$ & $172(92.5)$ & $32(86.5)$ & \\
\hline Antibacterial therapy & & & & 0.034 \\
\hline No & $39(17.5)$ & $37(19.9)$ & $2(5.4)$ & \\
\hline Yes & $184(82.5)$ & $149(80.1)$ & $35(94.6)$ & \\
\hline Hormone therapy & & & & $<0.001$ \\
\hline No & $128(57.4)$ & $119(64.0)$ & $9(24.3)$ & \\
\hline Yes & $95(42.6)$ & $67(36.0)$ & $28(75.7)$ & \\
\hline Immunoglobulin application & & & & 0.052 \\
\hline No & $151(67.7)$ & $131(70.4)$ & $20(54.1)$ & \\
\hline Yes & $72(32.3)$ & $55(29.6)$ & $17(45.9)$ & \\
\hline \multicolumn{4}{|c|}{ Traditional Chinese medicine treatment } & 0.129 \\
\hline No & $27(16.6)$ & $34(18.3)$ & $3(8.1)$ & \\
\hline Yes & $186(83.4)$ & $152(81.7)$ & $34(91.9)$ & \\
\hline
\end{tabular}

All variables with missing values are marked with a specific number of samples

Other symptoms include cough, expectoration, fatigue, headache, myalgia, sore throat, diarrhea, nausea, sneezing, nasal congestion, anorexia, night sweats, etc. Basic diseases include hypertension, diabetes, cardiovascular and cerebrovascular diseases, chronic obstructive pulmonary disease, chronic bronchitis, emphysema, chronic liver disease, chronic kidney disease, and neuropsychiatric diseases

$B M I$ body mass index, $P C T$ procalcitonin, $S B P$ systolic blood pressure, $D B P$ diastolic blood pressure, $W B C$ white blood cell, $A S T$ aspartate aminotransferase, $A L T$ alanine transaminase, $C R P$ C-reactive protein, $h s$ - $C R P$ hypersensitive c-reactive protein, $S A A$ Serum amyloid A, $P T$ prothrombin time, APTT activation of partial thrombin time

neutrophils, lymphocytes, direct bilirubin and indirect bilirubin (Table S1). In addition, more patients received antibacterial treatment $(94.6 \%$ vs. $80.1 \%)$ and glucocorticoid therapy (75.7\% vs. $36.0 \%)$ in the non-survivors than in the survivors, with $p$ values of 0.034 and $<0.001$, respectively (Table 1 ).

\section{The difference in the short-time survival among different cancer patients with SARS-CoV-2 infection}

Nearly $18 \%$ of the patients were lung cancer patients $(n=39)$, ranking first among all the 223 cancer patients with SARS-CoV-2 infection (Fig. 1a). Similarly, lung cancer patients accounted for $21.6 \%(n=8)$, which was the highest proportion in 37 non-survivors (Fig. 1b). To further explore the differences in short-term survival among patients with different tumor types, we conducted a Kaplan-Meier survival analysis (Fig. 1c). We found that lung cancer patients and other solid tumors patients had a better prognosis than hematological malignancies patients (the $p$ values were 0.031 and $<0.001$, respectively). Further a univariate Cox analysis showed the same results (Fig. 1d). This finding was also confirmed in multivariate Cox analysis after adjusting for age, gender, fever and dyspnea (Fig. 1e).

\section{The univariate and multivariate Cox analysis}

To explore the risk factors associated with SARS-CoV-2 infection in cancer patients, univariate and multivariate survival analysis were performed based on the Cox proportional hazard model. We randomly divided the 223 patients into a development cohort and a validation cohort according to a ratio of 


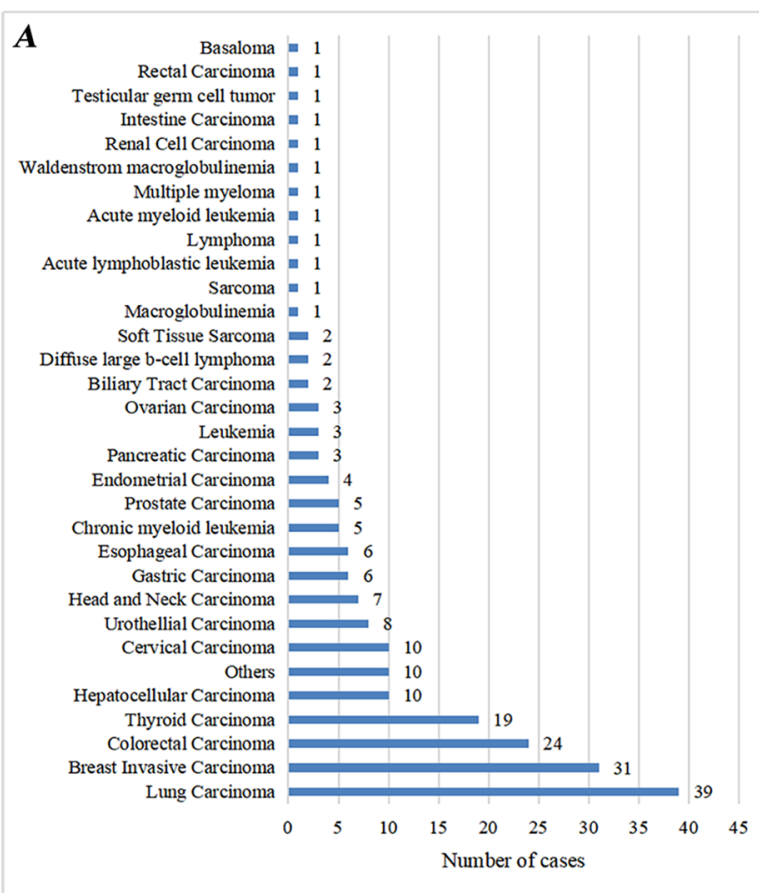

$\boldsymbol{B}$

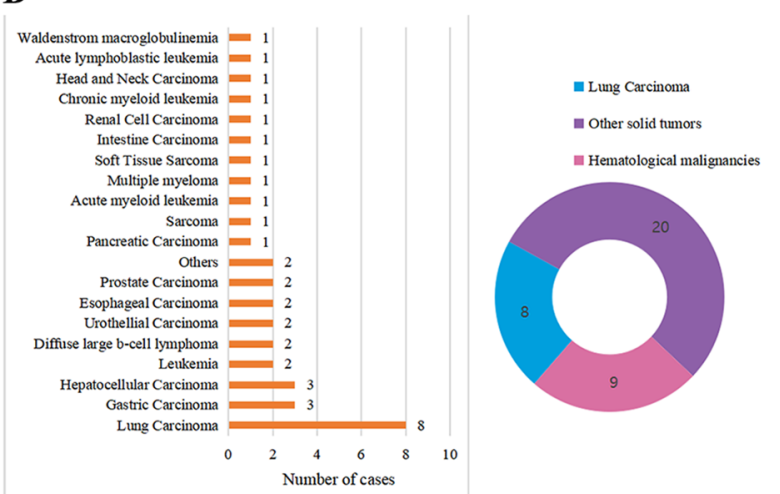

Fig. 1 a The number of cases with different tumor types in the 223 cancer patients with SARS-CoV-2 infection. b Distribution of tumor types in 37 non-survivors. There were 8 cases of lung cancer, 9 cases of hematological malignancies and 20 cases of other solid tumors. c Kaplan-Meier survival curves of hematological malignancies patients, lung cancer patients and other solid tumors patients infected

7:3. Table 2 shows the distribution of the clinical features of the two groups. There was no statistical difference in most clinical characteristics between the two groups, indicating that the baseline levels of the two groups were consistent. With the development cohort data, we performed a univariate Cox analysis of 24 clinical variables and found that gender, dyspnea, PCT, heart rate, white blood cell count, platelet count, neutrophil count, AST, direct bilirubin, total bilirubin, and D-dimer affected patient outcomes (Table 3). Using the mean or median of the continuous variables as the cut-off point, the Kaplan-Meier survival analysis was also performed on the 24 variables. Consistent with the results of univariate Cox
C

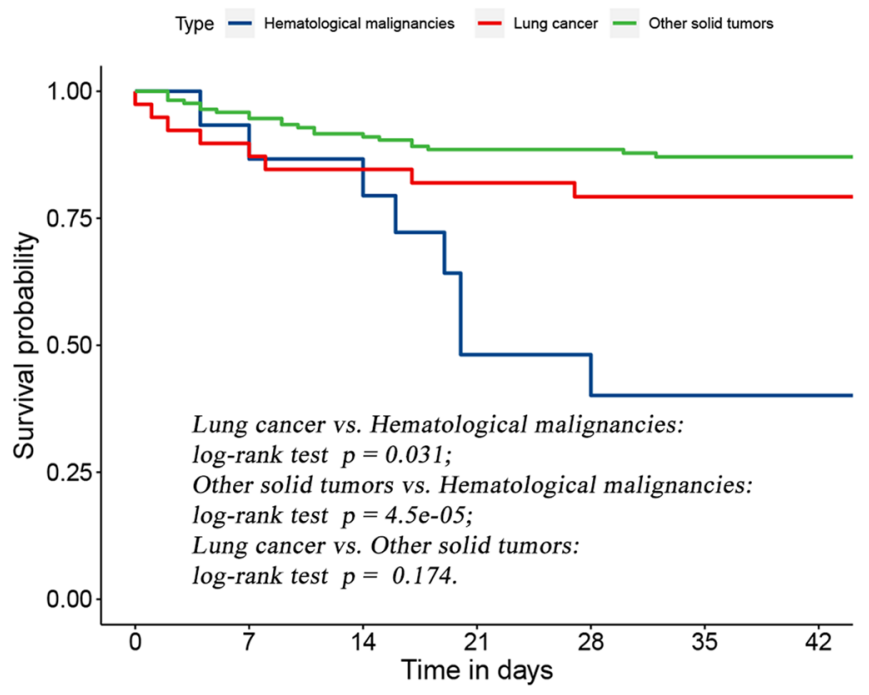

\begin{tabular}{|c|c|c|c|c|c|c|}
\hline \multicolumn{7}{|c|}{ Number at risk } \\
\hline 15 & 14 & 12 & 6 & 6 & 5 & 1 \\
\hline 39 & 35 & 32 & 31 & 29 & 25 & 5 \\
\hline 169 & 159 & 150 & 141 & 131 & 108 & 24 \\
\hline
\end{tabular}

$\boldsymbol{D}$

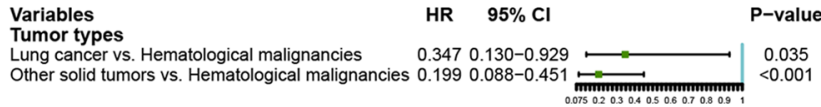

$\boldsymbol{E}$

\begin{tabular}{|c|c|c|}
\hline $\begin{array}{l}\text { Variables } \\
\text { Tumor types }\end{array}$ & $95 \% \mathrm{Cl}$ & P-value \\
\hline Lung cancer vs. Hematological malignancies & $0.2630 .095-0.729=$ & 0.011 \\
\hline Other solid tumors vs. Hematological malignancies & $0.2120 .091-0.493=$ & $<0.001$ \\
\hline Age & $1.0090 .981-1.038$ & 0.552 \\
\hline Sex (Male vs. Female) & $2.0010 .950-4.213$ & 0.068 \\
\hline Fever (With vs. Without) & $0.6630 .289-1.518=$ & 0.331 \\
\hline Dyspnea (With vs. Without) & $4.6092 .138-9.935$ & $<0.001$ \\
\hline
\end{tabular}

with SARS-CoV-2. Among cancer patients infected with SARS$\mathrm{CoV}-2$, compared with patients with solid tumors such as lung cancer, patients with hematological malignancies had a worse prognosis. d Univariate Cox analysis of different tumor types. e Multivariate Cox analysis after adjusting for age, gender, fever and dyspnea

analysis, gender, dyspnea, PCT, heart rate, platelet count, and D-dimer were also considered as prognostic factors (Figure S2). Moreover, the multivariate Cox analysis included the variables with $p<0.05$ in the univariate Cox analysis, where platelet count $(\mathrm{HR}=0.991, p=0.007)$ and neutrophil count (HR $=1.047, p=0008)$ were considered independent prognostic factors in these patients. 
Table 2 Clinical characteristics of SARS-CoV-2 infected cancer patients in the development and validation cohorts

\begin{tabular}{|c|c|c|c|}
\hline Variables & $\begin{array}{l}\text { Development cohort } \\
(n=159)\end{array}$ & Validation cohort $(n=64)$ & $p$ value \\
\hline Age (years) & $62(55,70)$ & $64(57,73)$ & 0.263 \\
\hline Sex & & & 0.272 \\
\hline Female & $80(50.3)$ & $27(42.2)$ & \\
\hline Male & 79 (49.7) & $37(57.8)$ & \\
\hline $\operatorname{BMI}\left(\mathrm{kg} / \mathrm{m}^{2}\right)$ & & & 0.323 \\
\hline$<18.5$ & $8(5.0)$ & $5(7.8)$ & \\
\hline $18.5-24.9$ & $57(35.8)$ & $16(25.0)$ & \\
\hline$\geq 25$ & 19 (11.9) & $11(17.2)$ & \\
\hline Unknown & $75(47.3)$ & $32(50.0)$ & \\
\hline Smoke & & & 0.143 \\
\hline Never & $117(73.5)$ & $53(82.8)$ & \\
\hline Previous/present & $42(26.5)$ & $11(17.2)$ & \\
\hline Anti-tumor therapy & & & 0.476 \\
\hline Continous & $93(58.5)$ & $33(51.6)$ & \\
\hline Discontinous & $22(13.8)$ & $8(12.5)$ & \\
\hline Unknown & $44(27.7)$ & $23(35.9)$ & \\
\hline Basic diseases & & & 0.069 \\
\hline Without & $81(50.9)$ & $24(37.5)$ & \\
\hline With & $78(49.1)$ & $40(62.5)$ & \\
\hline Fever & & & 0.863 \\
\hline Without & $39(24.5)$ & $15(23.4)$ & \\
\hline With & $120(75.5)$ & 49 (76.6) & \\
\hline Dyspnea & & & 0.724 \\
\hline Without & $96(60.3)$ & $37(57.8)$ & \\
\hline With & $63(39.7)$ & $27(42.2)$ & \\
\hline PCT (ng/ml) & & & 0.114 \\
\hline$\leq 0.5$ & $111(69.8)$ & $52(81.2)$ & \\
\hline$>0.5$ & $22(13.8)$ & $8(12.5)$ & \\
\hline Not application & $26(16.4)$ & $4(6.3)$ & \\
\hline Heart rate (bpm) & $88(77,100)$ & $87.5(78,100)$ & 0.829 \\
\hline $\mathrm{SBP}(\mathrm{mmHg})$ & $129.7(18.58)$ & $131.1(19.09)$ & 0.622 \\
\hline $\mathrm{DBP}(\mathrm{mmHg})$ & $78(70,85)$ & $80(71,85)$ & 0.895 \\
\hline Respiratory rate (braths/min) & $20(19,22)$ & $20(20,22.75)$ & 0.267 \\
\hline Temperature $\left({ }^{\circ} \mathrm{C}\right)$ & $36.7(36.5,37.5)$ & $36.8(36.5,38)$ & 0.798 \\
\hline WBC count $(10 E 9 / \mathrm{L})$ & $5.1(3.8,7.08)$ & $5.65(4.385,7.215)$ & 0.048 \\
\hline Neutrophil count $(10 E 9 / \mathrm{L})$ & $3.35(2.48,5.32)$ & $4.27(3.01,5.858)$ & 0.010 \\
\hline Lymphocyte count $(10 E 9 / \mathrm{L})$ & $0.88(0.62,1.44)$ & $0.96(0.553,1.275)$ & 0.892 \\
\hline Platelet count $(10 E 9 / \mathrm{L})$ & $186.8(96.71)$ & $200(89.69)$ & 0.340 \\
\hline $\operatorname{ALT}(\mathrm{U} / \mathrm{L})$ & $21(12,35)$ & $25(15.25,38.75)$ & 0.120 \\
\hline AST (U/L) & $26(18,38)$ & $32.5(22,44)$ & 0.013 \\
\hline Total bilirubin (umol/L) & $11.2(7.9,14.8)$ & $10.85(8.475,14.15)$ & 0.731 \\
\hline Direct bilirubin (umol/L) & $3.9(2.7,5.6)$ & $3.95(2.8,5.3)$ & 0.914 \\
\hline Creatinine (umol/L) & $63(51,82)$ & $71.5(56,92.75)$ & 0.022 \\
\hline D-dimer $(\mathrm{mg} / \mathrm{L})$ & $0.86(0.4,2.14)$ & $0.74(0.33,1.848)$ & 0.544 \\
\hline
\end{tabular}

$B M I$ body mass index, $P C T$ procalcitonin, $S B P$ systolic blood pressure, $D B P$ diastolic blood pressure, $W B C$ white blood cell, $A S T$ aspartate aminotransferase, $A L T$ alanine transaminase 
Table 3 Univariate and multivariate COX analysis of prognosis in the development cohort

\begin{tabular}{|c|c|c|c|c|}
\hline \multirow[t]{2}{*}{ Variables } & \multicolumn{2}{|l|}{ Univariate Cox analysis } & \multicolumn{2}{|c|}{ Multivariate Cox analysis } \\
\hline & $\mathrm{HR}(95 \% \mathrm{CI})$ & $p$ value & HR $(95 \%$ CI $)$ & $p$ value \\
\hline Age (years) & $1.017(0.983-1.053)$ & 0.332 & & \\
\hline Sex (male vs. female) & $2.513(1.042-6.059)$ & 0.042 & $2.996(0.861-10.43)$ & 0.085 \\
\hline \multicolumn{5}{|l|}{ BMI } \\
\hline $18.5-24.9$ vs. $<18.5$ & $0.965(0.119-7.846)$ & 0.973 & & \\
\hline$\geq 25$ vs. $<18.5$ & $0.777(0.070-8.578)$ & 0.837 & & \\
\hline Unknown vs. $<18.5$ & $1.491(0.671-11.34)$ & 0.700 & & \\
\hline Smoke (previous/present vs. never) & $1.405(0.601-3.283)$ & 0.432 & & \\
\hline \multicolumn{5}{|l|}{ Anti-tumor therapy } \\
\hline Discontinous vs. continous & $2.313(0.432-6.769)$ & 0.126 & & \\
\hline Unknown vs. continous & $2.020(0.495-4.972)$ & 0.126 & & \\
\hline \multicolumn{5}{|l|}{ Basic_diseases } \\
\hline With vs. without & $1.217(0.821-2.718)$ & 0.631 & & \\
\hline Fever (with vs. without) & $0.933(1.072-2.350)$ & 0.883 & & \\
\hline Dyspnea (with vs. without) & $5.044(0.198-12.71)$ & 0.001 & $2.942(0.934-9.266)$ & 0.065 \\
\hline \multicolumn{5}{|l|}{ PCT } \\
\hline$>0.5$ vs. $\leq 0.5$ & $6.316(0.158-15.26)$ & 0.000 & $1.789(0.598-5.348)$ & 0.298 \\
\hline Not application vs. $\leq 0.5$ & $1.824(0.548-5.816)$ & 0.310 & $1.619(0.445-5.887)$ & 0.465 \\
\hline Heart rate & $1.051(1.024-1.079)$ & 0.000 & $1.018(0.987-1.050)$ & 0.264 \\
\hline SBP & $0.993(0.971-1.016)$ & 0.545 & & \\
\hline DBP & $0.972(0.936-1.008)$ & 0.129 & & \\
\hline Respiratory rate & $1.034(0.939-1.140)$ & 0.496 & & \\
\hline Temperature & $0.949(0.577-1.554)$ & 0.828 & & \\
\hline WBC count & $1.177(1.063-1.305)$ & 0.002 & $1.115(0.947-1.312)$ & 0.192 \\
\hline Neutrophil count & $1.035(1.012-1.059)$ & 0.003 & $1.047(1.012-1.084)$ & 0.008 \\
\hline Lymphocyte count & $1.571(0.248-1.313)$ & 0.187 & & \\
\hline Platelet count & $0.993(0.988-0.998)$ & 0.006 & $0.991(0.984-0.997)$ & 0.007 \\
\hline ALT & $0.993(0.973-1.013)$ & 0.500 & & \\
\hline AST & $1.008(1.001-1.014)$ & 0.021 & $1.010(0.998-1.020)$ & 0.094 \\
\hline Total bilirubin & $1.031(1.014-1.048)$ & 0.000 & $0.961(0.872-1.058)$ & 0.413 \\
\hline Direct bilirubin & $1.043(1.022-1.065)$ & 0.000 & $1.071(0.938-1.223)$ & 0.314 \\
\hline Creatinine & $1.001(0.999-1.003)$ & 0.146 & & \\
\hline D-dimer & $1.037(1.007-1.067)$ & 0.014 & $1.026(0.985-1.068)$ & 0.225 \\
\hline
\end{tabular}

\section{Construction and evaluation of the prognostic nomogram}

A nomogram was constructed based on the final multivariate Cox analysis. Five factors closely related to prognosis were included in this model: sex, dyspnea, platelet count, neutrophil count and AST. According to the multivariate Cox analysis, each factor (in the nomogram) was assigned a score. We obtained the total score of nomogram from the sum of individual scores of all predictors. Based on the total score, patients' survival at 2, 3, and 5 weeks could be calculated by projection downward (Fig. 2). In this study, the C-index was 0.841 (95\% CI 0.782-0.900) in the development cohort and was 0.780 (95\% CI 0.678-0.882) in the validation cohort, indicating that the constructed model had reliable prediction performance. From the calibration curves of the development cohort (Fig. 3a-c) and the verification cohort (Figure S3a-c), it can be seen that the predicted value of the model is in good agreement with the observed value. Moreover, the clinical decision curves of the development (Fig. 3d-f) and validation (Figure S3d-f) cohorts for 2, 3, and 5 weeks also suggested that this nomogram had good clinical application significance.

\section{Development of an online tool to facilitate the clinical application of our constructed model}

To make it easier for clinicians to use this model, we created a dynamic web version of nomogram (https://covid-19-predi ction-tool.shinyapps.io/DynNomapp/). The interfaces of this web version are shown in Figure S4. On the right side of the interfaces, by inputting the corresponding data of patients 
Points

Sex

Dyspnea

Neutrophil Count

Platelet Count

AST

Total Points

2-week Survival

3-week Survival

5-week Survival

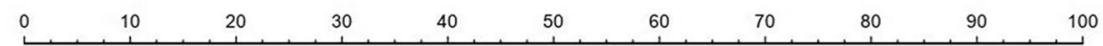

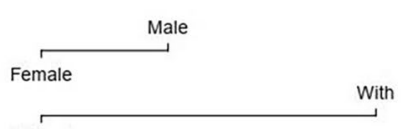

Without

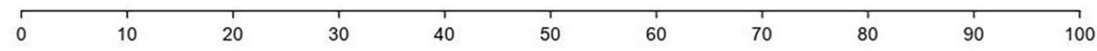

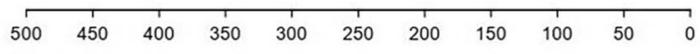

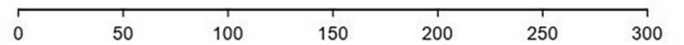

$20 \quad 40$

60

80

100

120

140

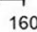

$\begin{array}{llllllllll}0.95 & 0.9 & 0.8 & 0.7 & 0.6 & 0.5 & 0.40 .3 & 1 & 1 & 1 \\ 1 & 0.2 & 0.1\end{array}$

$\begin{array}{lllllllll}0.95 & 0.9 & 0.8 & 0.7 & 0.6 & 0.50 .40 .30 .2 & 0.1\end{array}$

$\begin{array}{lllllllll}0.95 & 0.9 & 0.8 & 0.7 & 0.6 & 0.50 .4 & 0.30 .2 & 0.1\end{array}$
Fig. 2 A prognostic nomogram including significant clinical parameters for 2-week, 3-week, and 5-week OS in cancer patients with SARS-CoV-2 infection. By adding the scores obtained by projecting the corresponding 'Points' of each variable to the 'Total Point' axis, the total score can correspond to the corresponding prediction results
A

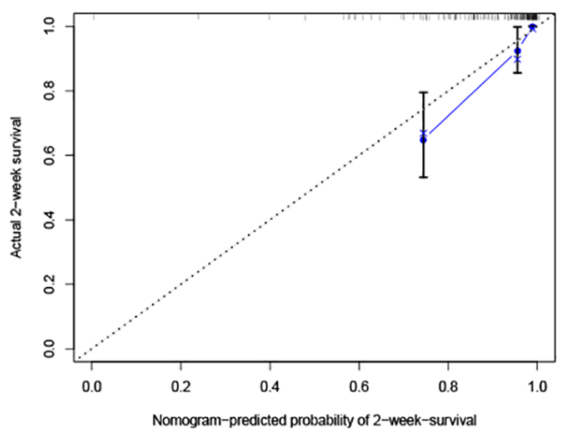

$D$

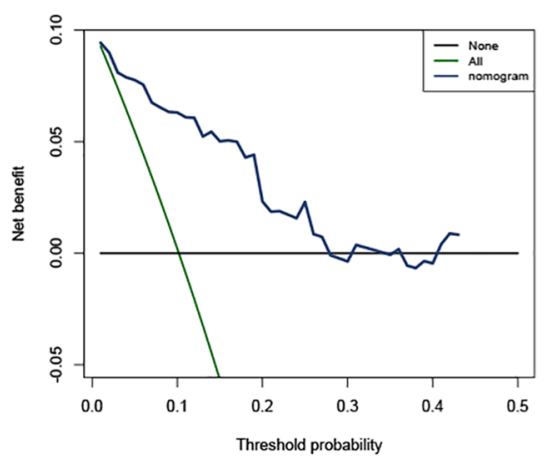

$B$

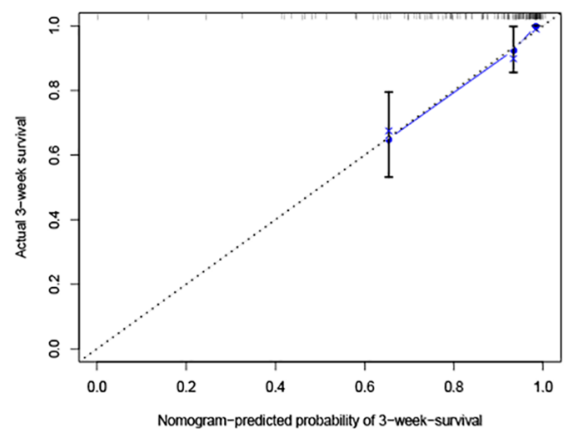

E

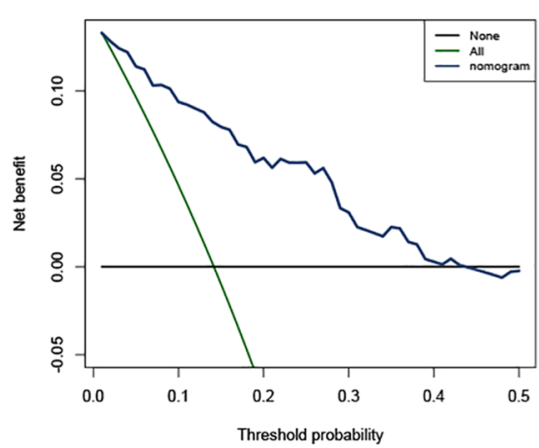

$C$

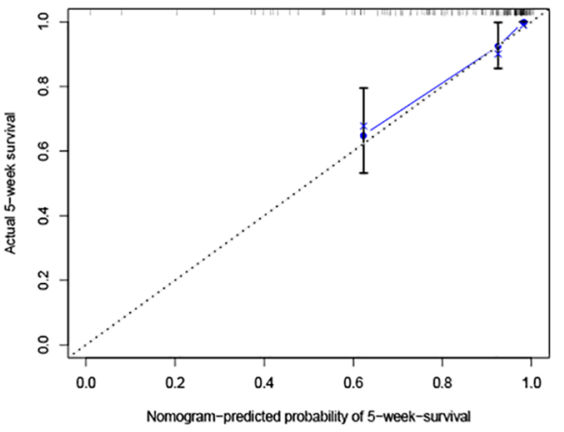

$F$

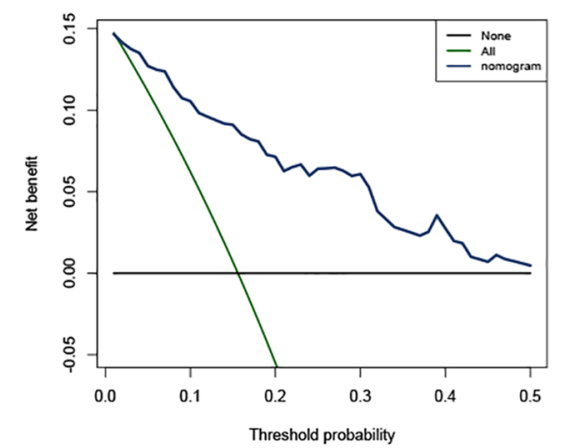

Fig. 3 Construction and evaluation of the prognostic nomogram. Calibration curves for 2-week (a), 3-week (b) and 5-week (c) OS in the development cohort. It could be seen that all calibration curves are close to the ideal $45^{\circ}$ dotted line. This indicates that the predicted value of the model had good consistency with the actual observed value. DCA curves for 2-week (d), 3-week (e) and 5-week (f) OS in the development cohort. In a large range of threshold probability, the net benefit of patients is higher than that of other two extreme cases (all and none), which shows that the nomogram model has good clinical applicability 
and clicking the button at the top of the page, the results would appear. Next, we could obtain the corresponding survival curves, survival probabilities of 2, 3 and 5 weeks and 95\% confidence intervals.

\section{Discussion}

Although some studies have confirmed that cancer patients were more susceptible to SARS-CoV-2 than the general population (Dai et al. 2020; Liang et al. 2020), the clinical features and short-term prognosis of cancer patients infected with SARS-CoV-2 were still unclear. This study showed that compared to survivors, non-survivors were mostly male and the elder, most of whom suffered from basic diseases and dyspnea. Moreover, the heart rate of the non-survivors was faster than that of the survivors, and the detection values of non-survivors were higher in most laboratory findings, such as white blood cell count, neutrophil count, PCT, CRP, hsCRP, direct bilirubin, PT, APTT, creatinine, CK-MB, and D-dimer, except for platelet counts and lymphocyte counts. These findings were consistent with previous researches on patients with SARS-CoV-2 infection (Chen et al. 2020a, b; Yang et al. 2020). Although cancer patients infected with SARA-CoV-2 shared common epidemiological characteristics with the general population, they might also have unique clinical features. Thus, this study performed further exploration of cancer patients infected with SARS-CoV-2 to evaluate the potential impact of COVID-19 on cancer patients.

The study of Dai et al. has demonstrated that patients with lung cancer were at the highest risk among patients with solid tumors infected with SARS-CoV-2 (Dai et al. 2020). They suspected that reduced pulmonary reserve and severe infection were responsible for poor outcomes in these patients. In this study, lung cancer patients accounted for the highest proportion of all cancer patients with SARS-CoV-2 infection. Similarly, lung cancer patients remained the most in 37 non-survivors, which might be associated with the high prevalence of lung cancer. The lung is the leading site of SARS-CoV-2 infection-induced lesions, and it remains unclear whether lung cancer patients have a worse prognosis than other cancer patients during the COVID-19 epidemic. In view of this, we divided these patients into hematological malignancies group, lung cancer group and other solid tumors group. Kaplan-Meier survival analysis, univariate and multivariate Cox analysis showed that the prognosis of patients with hematological malignancies was worse than that of patients with lung cancer and other solid tumors. Hematological malignancies in our study included leukemia, diffuse large B-cell lymphoma, acute lymphoblastic leukemia, acute myeloid leukemia, multiple myeloma, waldenström macroglobulinemia, and chronic myeloid leukemia. Because the immune function of malignant or dysfunctional plasma cells, lymphocytes or leukocytes in hematological malignancies was reduced (Lainey et al. 2013; Raab et al. 2009), all hematological malignancies patients were prone to severe infection complications, which might be the main reason for the high mortality rate of hematological cancer patients.

This study revealed that more patients were treated with antibacterial therapy and glucocorticoid therapy in the non-survivors than in the survivors, suggesting that nonsurvivors were often associated with bacterial infections and often accompanied by severe symptoms. Higher PCT values and neutrophil count for non-survivors also appeared to indicate this. PCT, the procalcitonin peptide synthesized from thyroid C cells and released from leukocytes, is a highly specific indicator of bacterial infection and is closely related to the prognosis of the disease (de Jong et al. 2016). In this study, PCT values of non-survivors were significantly higher than that of survivors, and PCT significantly affected the prognosis of patients. This was in line with the study of Chen et al. (2020a, b). Notably, although the participants of this study (cancer patients with SARS-CoV-2 infection) was different from that of Chen et al. (all patients with COVID19) (Chen et al. 2020a, b), PCT was considered to significantly affect patient outcomes in both studies. In addition, as the first defense barrier against suppurative infection, neutrophils play an essential role in the defense and protection function of the human body and are increased significantly in patients with bacterial infections. In this study, the median neutrophil count of non-survivors was $6.62 * 10 E 9$, significantly higher than that of the survivors $(3.42 * 10 E 9)$. These findings further suggested that patients with bacterial infections were at a higher risk of death and required more attention from clinicians.

Of the 223 patients included in this study, 133 patients had dyspnea and up to $75.7 \%$ of the 37 non-survivors had dyspnea. If not correctly managed, dyspnea may aggravate hypoxia and lead to acute respiratory failure and other serious complications. In this study, dyspnea was identified to affect the prognosis of patients in univariate Cox analysis and Kaplan-Meier survival analysis. As with dyspnea, sex, heart rate, platelet count, D-dimer, and AST were also demonstrated to be important factors affecting patient outcomes. Moreover, platelet count, as well as neutrophil count, was considered independent prognostic factors in these patients. These patients with low platelets had a higher risk of death, similar to ordinary COVID-19 patients (Bi et al. 2020; Lippi et al. 2020). The factors mentioned above could significantly affect the prognosis of patients, so clinicians should focus on the changes in these indicators when managing the patients with SARS-CoV-2 infection. We believe that sufficient identification of prognostic factors in such patients would help clinicians to determine the prognosis of patients in the early stage and adjust treatment strategies timely. 
Nomogram, a graphical prediction tool, can use statistical regression to assess the impact of various clinicopathological parameters on the likelihood of events occurring. Compared with the traditional staging system, nomogram has more accurate risk assessment methods, which is helpful to the proper individual treatment of clinical patients (Balachandran et al. 2015). According to the results of the multivariate Cox analysis, we also constructed a prognostic nomogram that accurately predicted the overall survival of patients at 2,3, and 5 weeks based on individual characteristics of patients. Importantly, the predictive performance and clinical utility of this model were also well-validated in an independent validation cohort. Furthermore, to facilitate clinicians to use the model established in this study, we created a dynamic nomogram on the web. Consequently, clinicians could access the website directly through mobile phone or computer anytime and anywhere, and inputting the corresponding information of patients to predict the survival of patients. This would undoubtedly simplify the application process and facilitate clinical use.

Indeed, this study has the following limitations. First, the nature of retrospective research inevitably led to selection bias. Second, despite our efforts to collect some essential clinical information after feedback, it was inevitable to omit some data that may inform our analysis. In addition, although the study used the patient's data from 26 clinical centers in Hubei province, Hubei was only one of the epicenter of the outbreak. It was still less representative than the cohort from the whole country or even several countries. Thus, it is still necessary to strengthen the in-depth cooperation among countries as soon as possible and carry out international large-scale or prospective researches.

In conclusion, this study provided evidence that male, dyspnea, elevated PCT, increased heart rate, elevated D-dimers, and decreased platelets were risk factors for cancer patients with SARS-CoV-2 infection. Abnormalities of a series of laboratory findings at admission were more common in non-survivors than survivors. Among cancer patients infected with SARS-CoV-2, compared with patients with solid tumors such as lung cancer, patients with hematological malignancies had a worse prognosis. In addition, the nomogram proposed in this study had good predictive performance, which would assist clinicians in predicting the prognosis of patients early and performing more reasonable and effective treatment strategies.

\footnotetext{
Author contributions Conception and design: WH, XL and CS. Collection and assembly of data: Data were collected by WH in collaboration with the authors. Data analysis and interpretation: WH, CS and XL. Manuscript writing: CS, HG and XD prepared the first draft of the report. WH, QS and YC co-supervised the project. Final approval of manuscript: WH had full access to all the data and had final responsibility to submit for publication.
}

Funding This study was supported by ZhongNan Hospital of Wuhan University Science Technology and Innovation Cultivating Fund (znpy2019088).

\section{Compliance with ethical standards}

Conflict of interest The authors have no conflict of interest to declare.

Ethical approval Editorial support was provided by the ethics committee of the National Cancer Center/Cancer Hospital, Chinese Academy of Medical Sciences and Peking Union Medical College (Approval No.: 20/061-2257).

\section{References}

Balachandran VP, Gonen M, Smith JJ, DeMatteo RP (2015) Nomograms in oncology: more than meets the eye. Lancet Oncol 16(4):e173-e180. https://doi.org/10.1016/S1470 -2045(14)71116-7

Bi XJ, Su ZX, Yan HX, Du JP, Wang JJ, Chen LP et al (2020) Prediction of severe illness due to COVID-19 based on an analysis of initial Fibrinogen to Albumin Ratio and Platelet count. Platelets 31(5):674-679. https://doi.org/10.1080/09537104.2020.17602 30

Chen NS, Zhou M, Dong X, Qu JM, Gong FY, Han Y et al (2020a) Epidemiological and clinical characteristics of 99 cases of 2019 novel coronavirus pneumonia in Wuhan, China: a descriptive study. Lancet 395(10223):507-513. https://doi.org/10.1016/ S0140-6736(20)30211-7

Chen RC, Liang WH, Jiang M, Guan WJ, Zhan C, Wang T et al (2020b) Medical Treatment Expert Group for COVID-19. Risk factors of fatal outcome in hospitalized subjects with coronavirus disease 2019 from a nationwide analysis in China. Chest 158(1):97-105. https://doi.org/10.1016/j.chest.2020.04.010

Dai MY, Liu DB, Liu M, Zhou FX, Li GL, Chen Z et al (2020) Patients with cancer appear more vulnerable to SARS-CoV-2: a multicenter study during the COVID-19 outbreak. Cancer Discov 10(6):783-791. https://doi.org/10.1158/2159-8290. CD-20-0422

de Jong E, van Oers JA, Beishuizen A, Vos P, Vermeijden WJ, Haas LE et al (2016) Efficacy and safety of procalcitonin guidance in reducing the duration of antibiotic treatment in critically ill patients: a randomised, controlled, open-label trial. Lancet Infect Dis 16(7):819-827. https://doi.org/10.1016/S1473 -3099(16)00053-0

Lainey E, Wolfromm A, Sukkurwala AQ, Micol JB, Fenaux P, Galluzzi L et al (2013) EGFR inhibitors exacerbate differentiation and cell cycle arrest induced by retinoic acid and vitamin D3 in acute myeloid leukemia cells. Cell Cycle 12(18):2978-2991. https://doi.org/10.4161/cc.26016

Liang WH, Guan WJ, Chen RC, Wang W, Li JF, Xu K et al (2020) Cancer patients in SARS-CoV-2 infection: a nationwide analysis in China. Lancet Oncol 21(3):335-337. https://doi.org/10.1016/ S1470-2045(20)30096-6

Lippi G, Plebani M, Henry BM (2020) Thrombocytopenia is associated with severe coronavirus disease 2019 (COVID-19) infections: a meta-analysis. Clin Chim Acta 506:145-148. https:// doi.org/10.1016/j.cca.2020.03.022 
Raab MS, Podar K, Breitkreutz I, Richardson PG, Anderson KC (2009) Multiple myeloma. Lancet 374(9686):324-339. https:// doi.org/10.1016/S0140-6736(09)60221-X

Yang XB, Yu Y, Xu JQ, Shu HQ, Xia JA, Liu H et al (2020) Clinical course and outcomes of critically ill patients with SARS-CoV-2 pneumonia in Wuhan, China: a single-centered, retrospective, observational study. Lancet Respir Med 8(5):475-481. https:// doi.org/10.1016/S2213-2600(20)30079-5
Publisher's Note Springer Nature remains neutral with regard to jurisdictional claims in published maps and institutional affiliations.

\section{Affiliations}

\section{Congkuan Song ${ }^{1,2} \cdot$ Zhe Dong $^{1} \cdot$ Hongyun Gong $^{3} \cdot$ Xiao-Ping Liu $^{4} \cdot$ Xiaorong Dong ${ }^{5} \cdot$ Aifen Wang $^{6} \cdot$ Yuan Chen $^{7}$. Qibin Song ${ }^{3}$. Weidong $\mathrm{Hu}^{1,2} \mathbb{C}$}

1 Department of Thoracic Surgery, ZhongNan Hospital of Wuhan University, 169 Donghu Road, Wuhan 430071, Hubei, China

2 Hubei Key Laboratory of Tumor Biological Behaviors, Hubei Cancer Clinical Study Center, Wuhan, China

3 Cancer Center, Renmin Hospital of Wuhan University, 238 Jiefang Road, Wuchang District, Wuhan 430060, Hubei, China

4 Department of Urology, ZhongNan Hospital of Wuhan University, Wuhan, China
5 Department of Thoracic Oncology, Xiehe Hospital, Tongji Medical College, Huazhong University of Science and Technology, Wuhan, China

6 Department of Obstetrics and Gynecology, Zhangjiagang Hospital TCM, Nanjing University of Chinese Medicine, Nanjing, China

7 Department of Thoracic Oncology, Tongji Hospital, Tongji Medical College, Huazhong University of Science and Technology, 1095 Jiefang Avenue, Wuhan 430000, Hubei, China 